Aus dem Hospitale der isr. Gemeinde in Budapest.

\title{
Die Aktion des Quecksilbers auf das syphilitische Gewebe.
}

\section{Ein Versuch zur Lösung der Frage nach dem Wesen der spezifischen Therapie.}

Zweite Mitteilung.

Von

Dr. J. Justus,

ordinierender Arzt für Hautkrankheiten.

Bevor wir in eınem nächsten Artikel die Ergebnisse darlegen, welche uns die Fortführung unserer Nachforschungen (1) brachte, sei es erlaubt, kurz auf fünf inzwischen erschienene Arbeiten einzugehen. Dies ist umsomehr geboten, als selbe sich fast ausschließlich mit einer Nachprüfung meiner Methodik befassen und trotz einer angeblich exakten Befolgung zu fast übereinstimmender Ablehnung gelangen.

Pollio (2) erhielt bei Behandlung syphilitischer Papeln von 8 verschiedenen Patienten, die Quecksilber in verschiedenster Quantität erhalten hatten und bei denen eine lokale Quecksilberbehandlung peinlichst vermieden war, nach Fixierung in $\mathrm{ZnCl}_{2}$ - oder in $\mathrm{ZnSO}_{4}$ - Lösung und Behandlung in Schwefelhydrogenwasser durch mehrere Stunden einen bräunlichen Niederschlag in den Stückchen. Mikroskopisch waren in sämtlichen Papeln schollige Massen von brännlicher Farbe erkennbar. Die Menge dieser Niederschläge stand aber in keinem Verhältnisse zur Menge des therapeutisch verwendeten Quecksilbers. In den Organstückchen von einem mit $\mathrm{Hg}$ behandelten Pferde zeigten sich nach der beschriebenen Behandlung ebenfalls die geschilderten bräunlichen Klümpchen. Pollio konnte bier auch nachweisen, daß die Menge der bräunlichen Klümpchen dem größeren oder geringeren Hg-Gehalt der tierischen Gewebe nicht proportional war. Dieses Mißverhälnis ließ ibn nunmehr zweifeln, ob die letzteren überhaupt mit dem in den Geweben befindlichen $\mathrm{Hg}$ in Beziehung gebracht werden konnten. Er setzte also 
kleine Nieren- und Leberstückchen von sicher Hg-freien Kaninchen, ferner einige nicht syphilitische Papeln von sicher Hg-freien Patienten und etliche syphilitische Papeln von noch nie mit $\mathrm{Hg}$ behandelten Patienten der Finwirkung des $\mathrm{ZnCl}_{2}$ und des $\mathrm{H}_{2} \mathrm{~S}$ aus und entdeckte auch hier im mikroskopischen Bilde die erwähnten bräunlichen, scholligen Gebilde. Demzufolge kann er dieselben nicht mit der $\mathrm{Hg}$ - Anwesenheit in den Geweben ursächlich in Beziehung bringen.

Kassai (3) bringt in Entgegnung auf Pollios Einwendungen in Erinnerung, daß $\mathrm{H}_{2} \mathrm{~S}$ nur in saurer Lösung zur Trennung der schweren Metalle geeignet ist. Da Pollio nicht angibt, ob er das $\mathrm{H}_{2} \mathrm{~S}$-Wasser angesäuert habe, liegt die Mörlichkeit vor, daß mit dem neutralen Reagens das im Gewebe physiologischerweise enthaltene $\mathrm{E}$ ise n einen schwärzlichen Niederschlag gab. Um diese Supposition zu beweisen, vergiftete $\mathrm{Kassai}$ eine Maus mit $3 \mathrm{mg} \mathrm{HgCl}_{2}$, außerdem verarbeitete er auch in ganz gleicher Weise die Organe einer anderen Maus, die überhaupt kein $\mathrm{Hg}$ erhalten hatte. Ein und dasselbe Organ wurde in Stücke zerschnitten verwendet, also z. B. eine Niere in zwei Teile ge. spalten und nach der Fixierung mit Zinklösung die eine Hälfte in saures, die andere in neutrales $\mathrm{H}_{2} \mathrm{~S}$-Wasser gelegt. Die Organe beider Tiere zeigten in der neutralen Lösung eine braunschwarze Durchtränkung, so wie es Pollio beschreibt. In dem sauren $\mathrm{H}_{2} \mathrm{~S}$ zeigten nur die Sublimatorgane eine mehr-minder diffuse Graufärbung, während die $\mathrm{Hg}$-freien Organe ihre gelbliche Eigenfarbe unverändert bewahrten. Mikroskopisch kann man aus den Schnitten aus neutralem $\mathrm{H}_{2} \mathrm{~S}$ dasselbe Verhalten der schwarzen und braunen Schollen beobachten, welches Polli o beschrieb, and zwar sowohl an den Organen des mit $\mathrm{HgCl}_{2}$ behandelten, als auch an denen des Kontrolltieres. Wurden aber die Organe aus angesäuertem $\mathrm{H}_{2} \mathrm{~S}$ untersucht, so waren an den des unvergifteten Tieres überhaupt keine schwarzen oder braunen Schollen zu bemerken, dagegen in den Schnitten aus den Organen der Hg-Maus sehr deutlich zu konstatieren.

Um sich zu vergewissern, daß die braunen und schwarzen Schollen, wie sie in den mit neutralem $\mathrm{H}_{2} \mathrm{~S}$ behandelten Schnitten vorhanden sind, wenn auch das Tier quecksilberfrei blieh, aus Schwefeleisen bestehen, legte $\mathrm{Kassai}$ einige derselben in eine verdünnte HCl-Lösung. Nach kurzer Zeit entstand ein charakteristischer $\mathrm{H}_{2} \mathrm{~S}$-Geruch und verschwanden die bräunlichen Schollen vollständig. Kassai betrachtet daher die Niederschläge in den quecksilberfreien Organen als Ferrosulfid und unterwarf selbe der Eisenreaktion mit Kaliumferricyanid mit positivem Erfolge (Berlinerblau-Reaktion). Die Einlagerungen in den Schnitten des Quecksilbertieres lösten sich dagegen in verdünnter Säure absolut nicht, waren daher als $\mathrm{HgS-Niederschläge} \mathrm{zu} \mathrm{betrachten.}$

Richard Fischel(4) unterzog den von Kassai verteidigten, von Pollio nicht exakt widerlegten histochemischen Nachweis des $\mathrm{Hg}$ einer nochmaligen Nachprüfung. Er unterwarf hypertrophische Papeln syphilitischer Kranken, die mit einer vorschiedenen Anzahl Quecksilber- 
Injektionen behandelt wurden, dem Verfahren zum Nachweise des Quecksilbers und fand bei den ersten drei Patienten dasselbe Bild, welches wir als typisch beschrieben haben. Dagegen konnte er bei einem anderen Versuche nach 13, bezw. 20 und 24 Injektionen meist $1 / 2 \mathrm{~cm}^{3}$ $1 \% \mathrm{HgCl}_{2}$.Lösung in den ausgeschnittenen Papeln entweder nur Niederschläge im Epithel oder aber überhaupt nichts auffinden. Ja noch mehr! die Papel einer vorher überhaupt nicht behandelten Arbeiterin wies ein meiner Schilderung ganz entsprechendes Bild auf. Ferner untersuchte Fischel spitze Kondylome Hg-freier Patienten und fand, ob gesäuerter oder ungesäuerter $\mathrm{H}_{2} \mathrm{~S}$ zur Füllung diente, mächtige Niederschläge ganz den von mir als HgS beschriebenen ähnlich. Die Tierversuche gaben ein übereinstimmendes Resultat. Sowohl vom Kontrolltier, als auch von dem mit $\mathrm{HgCl}_{2}$-Injektionen vergifteten Tiere wurden in Leber- and Nierenstückchen, die in gleicher Weise nach Justus behandelt wurden, dem Aussehen und der Verteilung nach die gleichen Niederschläge gefunden.

Was nun die von mir angegebenen und von Kassa $\mathrm{i}$ abermals besonders hervorgehobenen Reaktionen zur Unterscheidung des eventuell entstandenen Eisensulfids von dem Merkurisulfid betrifft, so verhielten sich nach $\mathrm{Fischel}$ die einzelnen Präparate ganz verschieden. In einem Falle verschwanden in einigen Schnitten die Niederschläge teilweise, in anderen fast gar nicht in $10-15 \% \mathrm{HNO}_{3}$ (24 Stunden). In einem Falle sine $\mathrm{Hg}$ blieben die zellartigen Niederschläge trotz sechsstündiger $\mathrm{HNO}_{3}$-Einwirkung erhalten. In dem größten Teile der übrigen Objekte sind die Niederschläge in der Salpetersäure vollständig verschwunden. Und endlich bemerkt $F$ is chel zu der Angabe Kas sa is, daß die braunen Schollen in den aus neutralem $\mathrm{H}_{2} \mathrm{~S}$ gewonnenen Präparaten die Realtion auf Eisensulfid geben: "warum berichtet $\mathrm{K}$ assai nicht, ob die durch gesäuerten $\mathrm{H}_{2} \mathrm{~S}$ gegangenen Objekte des sublimatvergifteten Tieres, die ja auch braune Scholleu aufwiesen, der Eisenreaktion unterworfen wurden." F is c hel fordert, daß Reaktionen, die wie diese Eisenreaktion in den landläufigen Lehrbüchern, so in der gewiß auf Vollständigkeit Anspruch machenden Enzyklopädie der mikroskopischen Technik nicht angeführt sind, mit größerer Genauigkeit veröffentlicht werden sollten. Fischels Resultate waren trotz Variationen der Konzentration der Reagentien nicht eindeutig. Er kommt zu dem Schlusse, daß die Theorien, die Justus auf seinen Methoden aufgebaut hat, jetzt ibrer Grundlage entbehren.

Konrad Siebert (5) untersuchte Nieren von mit Sublimat vergifteten Mäusen und von quecksilberfreien Kontrolltieren nach der Einwirkung von stark mit Salzsäure angesäuertem $\mathrm{H}_{2} \mathrm{~S}$. Er konnte bei der folgenden mikroskopischen Untersuchung einen Unterschied zwischen den Organen des Sublimat-Tieres und der $\mathrm{Hg}$-freien Tiere nicht finden. E'benso ergaben Condylomata lata vor Beginn der Kur exzidiert und 2 weitere nach der 5 . und 10 . Injektion ausgeschnittene keine besonderen Unterschiede. Sämtliche Schnitte von Tieren oder Mensohen stammend, 
zeigten dasselbe Verhalten verdünnter Salpetersäure gegenüber. In etwa $1 / 2$ Stunde waren alle Niederschläge durch die verdünnte Säure hinweggelöst. Si ebert kommt zu dem Schlusse, daß die nach der Justus schen Methode des $\mathrm{Hg}$-Nachweises entstehenden Niederschläge in den Geweben keine Hg-Sulfide sind.

Eine viel eingehendere Arbeit lieferte $A \mathrm{lmk}$ w ist (6). Wir wollen hier vorerst nicht die von ihm angegebene Methode besprechen, sondern uns bloß an die Resultate halten, die in seinen Händen, auf $\mathrm{Hg}$-hältige Organe vergifteter Tiere angewendet, unsere Methode ergab.

In den Nieren quecksilbervergifteter Kaninchen konnte $A \mathrm{~lm}$ kw is $t$ einen schwärzlich-braunen Niederschlag erhalten, welcher unter dem Mikroskope grau-gelblich erscheint. Dagegen konnte Almkwist in den Nieren quecksilberfreier Tiere diese Niederschläge $n i c h t$ beobachten. Dasselbe gilt auch für den Darm der Tiere. Almkwist gibt seiner Ansicht dahin Ausdruck, daß die durch meine Methode hervorgebrachten Niederschläge aus HgS bestehen, doch bemerkt er zugleich als Mangel meiner Methode, das selbe nicht gut fixiert und empfiehlt daher seine eigene Methode, mit welcher wir uns weiter unten beschäftigen wollen.

Wir trachteten in dem bisher angeführten die Ansichten der Autoren möglichst mit ihren eigenen Ausdrücken wiederzugeben. Es ist wohl unnötig zu bemerken, daß die Divergenz der Anschaungen und Ergebnisse eine sehr auffallende ist. Es sei uns erlaubt, bevor wir auf die detaillierte Kritik der Arbeiten der einzelnen Forscher eingehen, uns zuerst mit der das Wesen des Streites enthaltenden Hauptfrage zu beschäftigen. Diese Frage ist in ihrer möglichst präzisen Form die folgende: Wenn man quecksilberhältige Organe nach meiner Methode mit Zinksalz fixiert und mit saurem $\mathrm{H}_{2} \mathrm{~S}$ behandelt, sind die so entstandenen Niederschläge als HgS auzufassen oder nicht?

Um zu entscheiden, ob ein Niederschlag aus HgS besteht, gibt uns die qualitative Chemie eine Anzahl Proben an die Hand. Wenn man mit saurem $\mathrm{H}_{2} \mathrm{~S}$ in einem quecksilberhältigen Medium einen schwarzen Niederschlag erhält, so kann man annehmen, daß derselbe aus $\mathrm{HgS}$ besteht. $\mathrm{Ob}$ diese Annahme richtig sei, ist durch Reaktionen, die für $\mathrm{HgS}$ charakteristisch sind, entscheidbar. Die auf $\mathrm{HgS}$ charakteristischen Reaktionen sind die folgenden:

1. Starke rote $\mathrm{HNO}_{3}$ greift den Niederschlag weder in der Kälte, noch erwärmt an. Man müßte die Säure auf $70-75^{\circ}$ längere Zeit erwärmen und selbe auch erneuern, damit der Niederschlag nur grau gefärbt erscheint, durch Bildung von ${ }_{2} \mathrm{HgS}$. $\mathrm{Hg}\left(\mathrm{NO}_{3}\right)_{2}$. $\mathrm{S}$ entstehen.

2. Löst sich in Chlor entwickelnden Gemischen, indem $\mathrm{HgCl}_{2}$ und

3. $\mathrm{HBr}$ entwickelt in konzentrierter Lösung $\mathrm{H}_{2} \mathrm{~S}$; löst $\mathrm{HgS}$ beim Erwärmen.

4. HJ löst in konzentrierter Lösung auch in der Kälte, diluiert nur beim Erwärmen. 
5. Alkalien und alkalische Erden lösen es.

6. In der Mischung von $\mathrm{HNO}_{3}$ und $\mathrm{HCl}$ löst es sich schon in der Kälte.

Um daher einen schwarzen Niederschlag für $\mathrm{HgS}$ annehmen zu können, muß derselbe die eben geschilderten Reaktionen geben.

In den nachfolgenden Versuchen wollen wir die Anwendungsweise mehrerer Reaktionen auf die in quecksilberhältigen Organen bei Anwendung meiner Methode erhältlichen Niederschläge beschreiben.

Erster Versuch. Eine weiße Maus wird durch $3 \mathrm{mg} \mathrm{HgCl}_{2}$ Injektion getötet. Die frischen Organe (Leber, Nieren, Darm und Milz) kommen auf 2 Tage in 18\% ige Zinkacetat-Lösung, hierauf auf 3 Tage in gesättigtes, mit $\mathrm{HNO}_{3}$ versetztes $\mathrm{H}_{2} \mathrm{~S}$. Die gelblich-braun gewordenen Organe werden nun in steigendem Alkohol gebärtet und nachdem sie möglichst hart und zäh geworden, fein zerkleinert und in auf etwa das 10fache verdünnte Königswasser versetzt. Sie verweilen hier bis zum nächsten Tage. Nach 24 Stunden hat sich die schwarz-braune Farbe des Organbreies ganz verloren. Nun wird die Lösung mehrmals flitriert und in das ganz klare Filtrat eine Kupferspirale eingelegt. Es ist rätlich vor Einlegen der Spirale die Lösung ein wenig zu erwärmen, um etwaiges überschüssiges Chlor zu vertreiben. Nach 24 Stunden wird die Spirale aus der Lösung genommen, mit Wasser, Alkohol und Äther gewaschen und auf den Boden einer engen Eprouvette gebracht. Falls man nun diesen Teil der Eprouvette bis zur beginnenden Rotglut erhitzt, so wird der entstandene Dampf sich als kaum sichtbarer Beschlag an den kälteren höheren Teilen niederschlagen. Ein Jodkörnchen auf den Grund der Eprouvette gebracht und ein wenig erwärmt füllt die Eprouvette mit violetten Jod-Dampf und es entstehen hierauf hie und da kaum sichtbare weißlich-gelbe Niederschläge, die im Verlaufe von 1-2 Stunden zu ganz ausgezeichnet bemerkbaren roten Flecken und Streifen sich verwandeln. (Auf schwarzer Unterlage zu betrachten.)

Aus diesem Versuche folgt, daß der schwarze Niederschlag in verdünntem Königswasser löslich ist (6. Reaktion) und daß die Lösung nach der gebräuchlichen Ludwig-F ür bringerschen Methode untersucht den Quecksillergehalt als rotes $\mathrm{HgJ}_{2}$ erkennen läßt.

$\mathrm{Zweiter} V$ ersuch. Ein Meerschweinchen wird durch Injektion von $2 \mathrm{og} \mathrm{HgCl}_{2}$ getötet. Aus dem Darmrohre wird ein etwa $10 \mathrm{~cm}$ langes Stück ausgeschnitten und so behandelt, wie im ersten Versuche. Das fixierte und fein zerkleinerte Organ wird aber nicht mit Königswasser behandelt, sondern in salzsäurehältiges Wasser gegeben, in welches man einige Messerspitzen voll $\mathrm{KaClO}_{3}$ gibt. Nach 24 Stunden hat sich der Niederschlag gelöst. In dem Filtrate ist das Quecksilber so wie beim ersten Versuche nachweisbar.

Dieser zweite Versuch beweist also, daß sich der gebildete schwarze Niederschlag in chlorhaltiger Flüssigkeit löst und daß diese Lösung quecksilberhältig ist. (2. Reaktion.) 
Dritter Versuch. Eine weiße Maus wird durch Injektion von $3 m g \mathrm{HgCl}_{2}$ getötet. Die Leber, die Milz, die Nieren und der Darmtraktus werden in $20 \%$ iger $\mathrm{ZnCl}_{2}$ fixiert, hierauf 3 Tage lang in saurem $\mathrm{H}_{2} \mathrm{~S}$ gehalten. Die Organe werden nunmehr fein zerkleinert und in eine kleine Kochflasche zur Hälfte mit etwa 10mal verdünntem $\mathrm{HNO}_{3}$ gefüllt überfübrt. Man koche nun unter Ersatz des verdampfenden Wassers durch etwa 2 Stunden. Die Organe verwandeln sich zu einer klaren Lösung, in welcher aber zahlreiche gelblich-braune Krümmelchen ungelöst bleiben. (1. Reaktion.) Filtriert man nun, wäscht den auf dem Filter zurückgebliebenen gelblich braunen Satz mehrmals mit Wasser, spült selben vom Filter in eine Porzellanschale und löst denselben hierauf in verdünntem Königswasser, so kann man nach der Lu dwig-Fü rbringerschen Methode, wie oben geschildert, das Quecksilber nachweisen.

Vierter Versuch. Die Organe einer mit $3 \mathrm{mg} \mathrm{HgCl}_{2}$ vergifteten Maus werden ebenso behandelt, wie im dritten Versuche, nur wird der Niederschlag nicht in Königswasser, sondern in mit $\mathrm{KaClO}_{3}$ versetztem verdünnten $\mathrm{HCl}$ gelöst. (2. Reaktion). Das Quecksilber ist ebenfalls mit der Ludwig - Fürbringer schen Methode nachweisbar.

Ich habe diesen vierten Versuch mehrfach repetiert, immer mit demselben Ergebnisse.

Wir müssen aus den eben angeführten Versuchen folgern:

Die nach meiner Methode in quecksilberhäligen Geweben erfolgten Niederschläge lösen sich nicht in $\mathrm{HnNO}_{3}$, da selbe a ch nach zweistündigem Kochen nicht verschwinden.

In Chlor entwickelnden Flüssigkeiten löst sioh der schwarze Niederschlag (erster und zweiter Versuch). In Königswasser ist der Niederschlag löslich und aus all diesen Lösungen ist das Quecksilber nach der LudwigFürbringerschen Methode als rotes $\mathrm{HgJ}_{2}$ erhältlich.

Als Resultat der angeführten Versuche glaube ich den Satz feststellen zu können: die nach meiner Methode in quecksilber hältigen Geweben erhaltenen schwarzen Niederschläge bestehen aus $\mathrm{HgS}$, und ganz bestimmt daraf hinzuweisen, daB die von den nachprüfenden Autoren geäuBerten $\mathrm{Zweifel} g a \mathrm{z}$ haltlos sind.

Unterziehen wir nun die Arbeiten der obzitierten 4 Autoren einzeln einer eingehenden Kritik und bestreben wir uns so weit als möglich auch die Quelle ihrer unhaltbaren Folgerungen zu finden.

Mit Pollios Arbeit hat sich sehon $\mathrm{Kassai}$ sehr eingehend beschäftigt, so daß ich nur weniges zu bemerken have. Er läßt zwar in $\mathrm{Sieberts}$ Arbeit als eine private Mitteilung einfließen, daß das $\mathrm{H}_{2} \mathrm{~S}$, welches er benützte, angesäuert wurde, doch hat er dieses Umstandes in seiner eigenen Arbeit keine Erwähnung getan. Es könnte daher scheinen, daß Kassais Folgerungen, welche auf der Annahme der neutralen Reaktion des $\mathrm{H}_{2} \mathrm{~S}$ begründet sind, ihrer Basis entbehren. Bei der 
Besprechung der Arbeit Sieberts werden wir auch hierauf zurückkommen. Dagegen kann Pollio nicht von dem Vorwurfe Kassais frelgesprochen werden, daß er keine einzige der zahlreichen Proben zur Identifizierung des HgS benützt habe, um festzustellen, ob der Nieder schlag, den er in quecksilberhältigen Geweben erhielt, wirklich $\mathrm{HgS}$ sei ferner daß er keine Reaktion anwandte, um sich zu vergewissern, ob die in quecksilberfreien Geweben erhaltenen Niederschläge mit den eben genannten identisch seien.

Eine weit eingehendere Arbeit lieferte $\mathrm{F}$ ischel, doch sind die durch seine Versuche erhaltenen Resultate untereinander nicht nur sehr different, sondern geradezu so widersprechend, daB sie einen jeden Unbefangenen stutzig machen müssen. Wie oben erwähnt, gaben die von den ersten 3 mit Quecksiỉber behandelten Patienten. stammenden Schnitte genau das Bild, welches wir in unseren Präparaten gefunden und beschrieben hatten. Dagegen fand er in einer zweiten Versuchsreihe entweder nur einen ganz geringen Niederschlag oder er erhielt gar keinen. Anstatt daf Fischel die Crsache dieses widersprechenden Ergebnisses in seiner eigenen Technik gesucht hätte, wendete er sich auch der Richtung zu, in welcher Pollio vorging und untersuchte quecksilberfreie Organe. Da er nun in denselben auch "Niederschläge" fand, folgert er kurzerhand, daß die in der ersten Serie erhaltenen nicht aus $\mathrm{HgS}$ bestanden hatten. Wir müssen diesen Irrtum $\mathrm{Fischels} \mathrm{umso-}$ mehr hervorheben, da wir in unserer Orjginal-Arbeit, ferner in dem Artikel $\mathrm{Kassais}$ ganz genau umschrieben hatten, wie man $\mathrm{HgS}$ von anderen Niederschlägen zu unterscheiden hat. Sehen wir nun, wie Fis chel diese Reaktionen angewendet hat. Er behauptet, daß sich die einzelnen Präparate sehr verschieden verhielten, falls er selbe in 10 bis $15 \%$ iger $\mathrm{HNO}_{3}$-Lösung 24 Stunden liegen ließ. In einem Falle lösten sich aus einigen Schnitten die Niederschläge zum Teile, in anderen verschwanden sie ganz. Wir müssen daraus folgern, daß die löslichen Niederschläge nicht aus HgS bestanden hatten.

Was die Reaktion auf Eisen anbelangt, erhebt $\mathbf{F i s c h e l}$ gegen Kassai den Vorwurf, letzterer hätte diese Reaktion nicht genau beschrieben und demzufolge wären $F$ ischels Versuche, obzwar er mit verschiedenen Konzentrationen Versuche machte, nicht eindeutig ausgefallen. Wir müssen diesen Vorwurf zurückweisen, denn die Probe auf Eisen im Gewebe ist seit den 1860er Jahren bekannt und wir fanden selbe ganz genau in nicht weniger als 5 bekannten Werken wiedergegeben. ${ }^{x}$ ) Wir müssen daher aus Fis chels Arbeit nicht die Folgerung ziehen, daß unsere Theorien ihre Grundlagen verloren hätten, sondern daB Fischel mit einer sehr mangelhaften Technik gearbeitet hat, der

1) Perls: Virchows Archiv Bd. XXXIX.

Joseph u. Löw en bach. Dermatohistologische Teehnik 1900. p. 43.

Ledermann. Mikr. Techmk 1903. pg. 107.

Friedländer Eberth. Mikr. Technik 1900. pag. 182. pag. 182

Enzyklopädie der mikroskop. Technik. Erster Band. 
zufolge er in quecksilherhältigen Geweben einmal den HgS-Niederschlag erbielt, das andere Mal nicht; ferner, daB er nicht im Stande war, den einfachen Umstand zu beurteilen, ob die Niederschläge, die er erhielt, in $\mathrm{HNO}_{3}$ löslich sind und endlich, daß er eine ganz allgemein bekannte, in den meisten Lehrbüchern enthaltene Probe übersah.

Noch mehr müssen wir den Vorwurf einer mangelhaften Technik Sieb ert gegenüber erheben. Er bekam die unzweideutigsten Ergebnisse und behauptet, daß weder mit freiem Auge noch durch mikroskopische Untersuchung ein Unterschied zwischen quecksilberhältigen und quecksilberfreien Organen zu finden gewesen. Si ebert folgert aus diesem Umstande, daß die nach der Justusschen Methode erhaltenen Niederschläge nicht aus HgS bestehen. Wir müssen auf Grund seiner Resultate ganz andere Folgerungen ziehen.

Er hatte die Methode sehr mangelhaft beherrscht und demzufolge weder in quecksilberhältigen, noch weniger in quecksilberfreien Geweben einen HgS-Niederschlag erhalten, sondern in beiden Eisen-Sulfid. Dies beweist Sieberts Behauptung, daß sich sämtliche Niederschläge in $\mathrm{HNO}_{3}$ lösten. Wir müssen ferner hier wieder hervorheben, daß auch $\mathrm{S}$ i ebe $\mathrm{rt}$ keinen einzigen Versuch daraufhin angestellt hat, ob die durch ihn erhaltenen Niederschläge nicht aus Eisen-Sulfid bestehen.

Unsere Kritik der oben genannten Arbeiten wäre nicht vollständig; wenn wir es nicht versuchen würden, den Umstand zu erklären, worin es begründet war, daß die Autoren in quecksilberhältigen Organen keinen HgS-Niederschlag bekamen. Die Antwort auf diese Frage liegt in der Art des Entstehens von $\mathrm{HgS}$.

Wenn man aus $\mathrm{HgCl}_{2}$ oder $\mathrm{HgNO}_{3}$-Lösung das Quecksilber in Form von $\mathrm{HgS}$ ausfällen will und zu diesem $\mathrm{Z}$ wecke $\mathrm{H}_{2} \mathrm{~S}$-Wasser hinzufügt, so erhält man in erster Reihe einen weißen Niederschlag. Dieser weiße Niederschlag wird nur bei weiterem Hinzufügen von $\mathrm{H}_{2} \mathrm{~S}$ gelblich, mit noch mehr $\mathrm{H}_{2} \mathrm{~S}$ braun und endlich schwarz.

Nach Treadwell entsteht der weiße Niederschlag wie folgt:

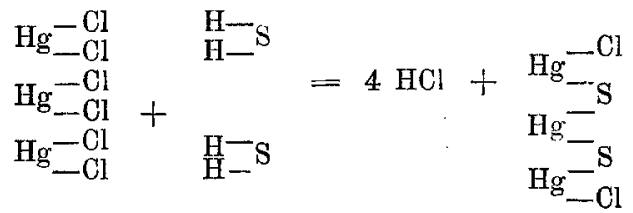
sulfid :

Bei weiterem Hinzufügen erhält man endlich schwarzes Merkuri-

$\mathrm{Hg}_{3} \mathrm{Cl}_{2} \mathrm{~S}_{2}+\mathrm{H}_{2} \mathrm{~S}=2 \mathrm{HCl}+3 \mathrm{HgS}$.

Hieraus folgt daher, daß wenn man nicht genügende Mengen $\mathrm{H}_{2} \mathrm{~S}$ angewendet hat, man keinen schwarzen, sondern einen weißen eventrell einen gelben, höchstens einen braunen Niederschlag erhalten wird. Wenn wir nun auch den Umstand in Betracht ziehen, $d a ß$ das im Gewebe enthaltene $\mathrm{FIg}$ für das in der Lösung enthaltene $\mathrm{H}_{2} \mathrm{~S}$-Gas schwerer erreichbar ist, so wird es erklärlich, daß die ungenügende Zeit oder nicht mit 
großen Mengen $\mathrm{H}_{2} \mathrm{~S}$ behandelten Gewebe entweder nur einen weißen oder höchstens einen gelben Niederschlag enthalten, doch keinen braunen oder schwarzen. Nun ist aber bei mikroskopischer Untersuchung in den hiezu geeigneten recht dünnen Schnitten weder der weiße, noch der gelbe Niederschlag wahrnehmbar, auch der braune nur schwer, gut ist nur der schwarze Niederschlag zu unterscheiden. Aus diesem ungenügenden Behandeln mit $\mathrm{H}_{2} \mathrm{~S}$ ist es daher zu erklären, dab $\mathrm{Fi}$ s chel nicht in jedem quecksilberhältigen Gewebe einen solchen schwarzen Niederschlag erhielt, der in $\mathrm{HNO}_{3}$ unlöslich war. Noch ungenügender hat $\mathrm{Siebert}$ seine Präparate mit $\mathrm{H}_{2} \mathrm{~S}$ behandelt, denn er erhielt überhaupt niemals einen in $\mathrm{HNO}_{3}$ unlöslichen Niederschlag.

Was die Niederschläge betrifft, die Fischel in quecksilberfreien Geweben erhielt, so bestanden sie zweifellos aus Eisen-Sulfid, ebenso wie die Niederschläge, die Siebert in quecksilberhältigen und quecksilberfreien Geweben gleichmäßig erhielt. Wenn man nämlieh zu einer Eisenlösung $\mathrm{H}_{2} \mathrm{~S}$ fügt, so entsteht sogleich ein schwärzlicher Niederschlag. Fis chel und $\mathrm{Si}$ ebert haben zwar saures $\mathrm{H}_{2} \mathrm{~S}$ angewandt, welches mit Eisen keinen Niederschlag gibt, doch haben sie vergessen, sich darüber Gewißheit zu verschaffen, daß nach Herausnahme aus dieser sauren Lösung auch alles $\mathrm{H}_{2} \mathrm{~S}$ aus dem Gewebe durch Auswaschen in säurehältigem Wasser entfernt wird, denn sonst bleibt das $\mathrm{H}_{2} \mathrm{~S}$ sebr lange im Gewebe haften (man verspürt seinen Geruch noch tagelang) und kann noch nachträglich in Alkohol oder sogar noch später in den Schnitten mit dem Eisen eine Eisen-Sulfid-Verbindung ergeben.

Eine eingehendere Besprechung wollen wir der sehr fleißigen und ausgedehnten Arbeit Alm kvists zu teil werden lassen, doch sei es erlaubt, vorerst auf die zu Beginn Kapitel 2 seines Werkes enthaltenen Äußerungen hier einzugehen. Almkvist äußert sich hier nämlich folgendermaßen. Er hätte im Mai 1901 seine ersten Versuche, eine Methode für die histochemische Reaktion des Quecksilbers im Gewebe zu finden, angestellt und im Augusthefte 1901 des Archivs für Dermat. und Syphil. sei meine Arbeit „die Aktion des Quecksilbers auf das syphilitische Gewebe" erschienen. Ich muB diese Behauptung A Im kvists dahin rektifizieren, daß meine ersten Vorträge über diesen Gegenstand schon im Jahre 1897 gehalten wurden, daß ich die ganz vollendete Methode schon auf dem internationalen Kongresse für Dermatologie in Paris im Jahre 1900 (7) veröfientlicht habe und demzufolge zur Zeit als A $1 \mathrm{mkvist}$ sich mit dem Gedanken einer histochemischen Reaktion des Quecksilbers zu befassen begann, die vollständig ansgearbeitete Methode an einem leicht zugänglichen Orte bereits veröffentlicht war.

Almkvist stellt im Kapitel 2 seiner Arbeit die Behauptung auf, $\mathrm{da} \beta$ das in $\mathrm{HgS}$-Albuminat gebundene Quecksilber durch $\mathrm{H}_{2} \mathrm{~S}$ fällbar ist.

Ich muß ihm auf Grund von Nachprüfungen in dieser Behauptung vollständig Recht geben und in dieser Beziehung das in meiner ersten Arbeit Gesag te zur ü knehmen. Wenn QuecksilberAlbuminate genügende Zeit mit genügenden Mengen $\mathrm{H}_{2} \mathrm{~S}$ in Berührung 
kommen, so entsteht HgS. Das Quecksilber in den Hg-Albuminaten verhält sich nämlich wie eine sehr wenig dissoziierte Verbindung. Nun ist es aber bekannt, daß auch eine minimal dissoziierte QuecksilberVerbindung wie z. B. das Hg-Cyanid mit Schwefelhydrogen einen schwarzen Niederschlag gibt, während es die anderen QuecksilberReaktionen nicht gibt. Wenn man daher die wenig dissoziierten Quecksilber-Albuminate unter günstigen Umständen mit überschüssigem $\mathrm{H}_{2} \mathrm{~S}$ behandelt, so wird man sämtliches $\mathrm{Hg}$ als $\mathrm{HgS}$ fällen können. Diese Anschaungen machen auch Almkvist s Methode verständlich. Dieselbe ist die folgende. Die ausgeschnittenen Stückchen kommen sogleich in

1. gesättigtes Schwefelhydrogenwasser, dem man unmittelbar vor der Anwendung soviel Salpetersäure zugesetzt hat, daß es ungefähr $4 \%$ $\mathrm{HNO}_{3}$ enthält. Hierin bleiben die Stückchen 8-10 Stunden, können jedoch, ohne Schaden zu nehmen, bis zu 48 Stunden darin liegen. Danach kommen die Stückchen in

2. Alkohol von steigenden Prozenten, sodann

3. Einbettung (Paraffin).

Almkvist behauptet, daß man mit dieser Methode das in den Geweben sublimatvergifteter Tiere enthaltene Quecksilber als HgS erhalten und außerdem die Gewebe in zufriedenstellender Weise fixieren kann. Daß dem so ist, beweist er durch Illustrationen, welche das makroskopische Aussehen so behandelter Nieren wiedergeben und durch mikroskopische Schnitte, wo in den Zellen das gelblich e HgS sichtbar ist. Außer den Nieren ist es Almkvist nur noch im Darme gelungen $\mathrm{HgS}$ nachzuweisen. Die anderen Organe betreffend zitieren wir seine Äußerung: „In allen anderen untersuchten Organen als den Nieren und dem Darme istes mir nichtgelungen, mikroskopisch das Quecksilber zu finden. Besonders hebe ich hervor, daß in der Leber, wo die chemische Analyse stets soviel Quecksilber nachgewiesen hat, nur Pigmentansammlungen, u. zw. oft in kolossalen Mengen, nie aber Schwefelquecksilber-Körnchen finden können, trotzdem man erwarten sollte, da man sich in den Speicheldrüsen eine Sekretion von Quecksilber vorgestellt hat, hier ähnliche HgS-Körnchen wie in den Nieren zu finden."

A lm kvist folgert daher, daß in diesen Organen sich kein anderes Quecksilber befinden könne, als dasjenige, welches im Blute, resp. in der Lymphe enthalten sei. Dieser seiner Auffassung muB ich entschiedenentgegentreten. Almkvistfand in den anderen Organen nur deshalb kein Hg, weil seine Methode nicht genügend ist, das in diesen Organen fest gebundene Quecksilber nacheuweisen, nicht aber, weil in diesen Organen kein $\mathrm{Hg}$ enthalten ist.

Almkvists Methode erlaubt nämlich in den Nieren auch nur das Darstellen eines gelblichen Niederschlages, ist daher mit anderen Worten nicht genügend, um bis zum braunen, resp. schwarzen HgS fortzuschreiten. Wenn wir daher nun in Betracht ziehen, daß das in den 
Nieren sekretierte Quecksilber nur in einer ganz losen chemischen Bindung vorhanden sein kann, was schon dadurch bewiesen wird, daß es im Harne mit der einfachsten Probe nachweisbar ist, ja sogar als reduziertes metallisches Quecksilber zu finden ist, und daß Almkvists Methode auch in den Nieren nur gelbliches $\mathrm{HgS}$ ergibt, so wird es natürlich erscheinen, daß dort, wo das Quecksilber stark in den Zellen gebunden ist, seine Methode keinen sichtbaren Niederschlag ergibt. Scheinbar würde gegen diese meine Auffassung der Umstand sprechen, $\mathrm{daB} A \operatorname{lmkvist}$ auch in der Darmwand einen Niederschlag erhielt, aber da gibt Alm kvist selbst za, daß das im lebenden Tiere im Darmrohre enthaltene $\mathrm{H}_{2} \mathrm{~S}$ die Fällung verursacht. Wir müssen daher Almkvists Behauptung, daß ausschließlich nur in den Nierenzellen Quecksilber sekretiert wird, dagegen in allen anderen Organen das Quecksilber in den Zellen nicht enthalten ist, und in dem Darme auch nur das in den Leukocyten enthaltene Quecksilber durch den natürlichen $\mathrm{H}_{2} \mathrm{~S}$ Gehalt gefällt wird, die Darm-Epithelien dagegen kein Quecksilber sekretieren, als vollständig unhaltbar bezeichnen.

Unsere hierauf bezüglichen Untersuchungen an Organen $\mathrm{Hg}$ vergifteter Tiere und Menschen angestellt, ergaben die Anwesenheit des Hg in den Zellen verschiedener Organe.

Wir wollen in einem nächsten Artikel Methodik und Ergebnisse detailliert veröffentlichen.

Die Zusammenfassung dieser gegenwärtigen Arbeit sei in folgenden Sätzen gegebea:

1. Die durch meine Methode erhaltenen Niederschläge in den Geweben $\mathrm{Hg}$ vergifteter Tiere oder im syphilitischen Gewebe quecksilberbehandelterKranken besteben aus Merkurisulfid.

2. Diese Niederschläge lassen ihre chemische Natur durcb die Anwendung zablreicher, für HgS charakteristischer Reaktionen zweifellos demonstrieren.

3. Die widersprechenden Ergebnisse von Pollio, Fischel, Siebert sind durch eine ungenügende Behandlung mit $\mathrm{H}_{2} \mathrm{~S}$, respektive durch eine lückenhaft a us eführte oder aberganzunterlassene Differenzierung von eventuell entstandenem Ferrosulfid hervorgerafen.

4. Die bedeutendere Zeit nach meiner Veröfentlichung angearbeitete Methode von Almkvist zum Nachweis des $\mathrm{Hg}$ in Geweben vergifteter Tiere erlaubt nur einen gewisen Anteil des $\mathrm{Hg}$ als $\mathrm{HgS}$ darzustellen, ist dalier für eine Lokalisierung des $\mathrm{Hg}$ in den Zellen selbst nicht brauchbar. 


\section{$L$ i t e ratur.}

1. Justus. Die Aktion des Quecksilbers auf das syphilit. Gewebe. Dieses Archiv. Bd. LVII.

2. Pollio. Über die Aktion etc. Dieses Archiv. Bd. LX.

3. Kassai. Über die Aktion etc. Dieses Archiv. Bd. LXII.

4. Fis chel. Über die Aktion etc. Dieses Archiv. Bd. LXVI.

5. Siebert. Über die Aktion etc. Dieses Archiv. Bd. LXVII.

6. A lmkvist. Nordiske medicinkst Ark. Heft 2. 1903.

7. Justus. Comment est-ce que la mercure guérit dans la syphil. IV. Congrès internat. de dermat. 